\title{
The climatology of ionospheric plasma bubbles and irregularities over Brazil
}

\author{
F. D. Chu ${ }^{1,2}$, J. Y. Liu $^{1,3}$, H. Takahashi ${ }^{4}$, J. H. A. Sobral ${ }^{4}$, M. J. Taylor ${ }^{5}$, and A. F. Medeiros ${ }^{6}$ \\ ${ }^{1}$ Institute of Space Science, National Central University, Taiwan \\ ${ }^{2}$ National Standard Time and Frequency Laboratory, Telecommunication Laboratories, Chunghwa Telecom Co. Ltd., Taiwan \\ ${ }^{3}$ Center for Space and Remote Sensing Research, National Central University, Taiwan \\ ${ }^{4}$ Instituto Nacional de Pesquisas Espaciais-NIPE, São José dos Campos, SP, Brazil \\ ${ }^{5}$ Space Dynamics Laboratory and Physics Department, Utah State University, USA \\ ${ }^{6}$ Departamento de Física, Universidade Federal de Campina Grande, Campina Grande, Brazil
}

Received: 6 October 2003 - Revised: 27 October 2004 - Accepted: 2 November 2004 - Published: 28 February 2005

\begin{abstract}
In this paper, we examine ionospheric plasma depletions (or bubbles) and irregularities observed by photometers and a ground-based receiver of the global positioning system (GPS) both located in Brazil, South America. A simultaneous observation reveals that when the ionospheric plasma becomes depleted, the GPS total electron content decreases and the associated phase fluctuations grow. Statistical results show that the plasma bubbles and the significant GPS phase fluctuations generally occur during OctoberMarch. The solar activity variation is not obvious in either observation except during the equinoctial months. The GPS phase fluctuations suggest that the most pronounced ionospheric irregularities appear between 20:00 and 01:00 LT, which generally agree with previous ionospheric bubble observations.
\end{abstract}

Key words. Ionosphere (equatorial ionosphere; ionospheric disturbances; ionospheric irregularities)

\section{Introduction}

Ionospheric plasma depletions (or bubbles) often coexist with ionospheric irregularities in the equatorial regions (Kelley, 1989). The plasma bubbles can be detected by means of optical techniques (Weber et al., 1978), as well as by scintillation techniques (Aarons, 1993). The first set of plasma bubble detections through the nocturnal OI6300 ̊̊ airglow observations was carried out by VanZandt and Peterson (1968) in Hawaii (geographical: $20.7^{\circ} \mathrm{N}, 156.3^{\circ} \mathrm{W}$; geomagnetic: $22^{\circ} \mathrm{N}$ ). They reported "enhancements in the form of northsouth ridges" of the OI6300 $\AA$ airglow but did not associate this with the bubble phenomenon. The nocturnal phenomenon of the large-scale ionospheric plasma bubbles has

Correspondence to: $\mathrm{J} . \mathrm{Y} . \mathrm{Liu}$

(jyliu@jupiter.ss.ncu.edu.tw) been extensively surveyed over Brazil for more than two decades through optical techniques (see the papers listed in Taylor et al., 1997; Sobral et al., 1980a, 1980b, 1999; Takahashi et al., 2001). Since the optical techniques only can be carried out during clear nights, a long-term observation is necessary. Based on data from 934 days of experimental observations of the atomic oxygen $630 \mathrm{~nm}(6300 \AA)$ nocturnal airglow, obtained by means of scanning photometers and imagers located at Cachoeira Paulista $\left(45.0^{\circ} \mathrm{W}, 22.7^{\circ} \mathrm{S}\right.$; $-16.7^{\circ} \mathrm{N}$ geomagnetic), Sobral et al. (2002) reported the occurrence characteristics of the large-scale ionospheric plasma depletions, during the period of 1977-1998, with solar activity, season and geomagnetic activity.

Over the past two decades, observations of the satellite signals have been used to derive phase and amplitude scintillation at various latitudes (see the papers listed in Aarons, 1993). A spaced-antenna scintillation system has been demonstrated that is able to establish climatology of zonal drift (Valladares et al., 1996). Furthermore, the establishment of a Global Positioning System (GPS) provides an alternative way to investigate the ionospheric irregularities. Some applications of GPS observations to equatorial aeronomy in South America have been reported (Aarons et al., 1997; Mendillo et al., 2000; Shan et al., 2002). Aarons et al. (1997) used GPS phase fluctuation, which is practically the time variation of the total electron content (TEC), as an indicator of the occurrence of ionospheric irregularities to study the occurrence of irregularities during the storm periods of 1993-1995. Mendillo et al. (2000) further developed two indices to quantitatively examine the GPS phase fluctuations in various months during 1995-1998.

A survey of the existing photometer/imager measurements (Sobral et al., 2002) and databases of the International GPS services (IGS) shows that there is some overlap between the coverage of the scanning photometers at Cachoeira Paulista and that of the ground-based GPS receiver at Brasilia 


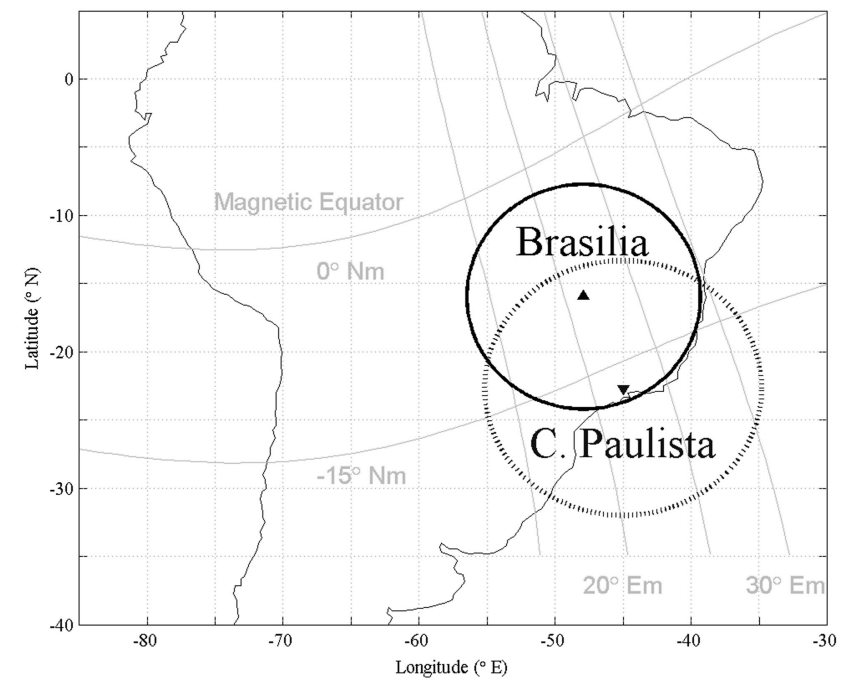

Fig. 1. The view area and encompassed coverage of the photometer at Cachoeira Paulista (dashed circle) and the GPS receiver at Brasilia (solid circle) superposed on the map of South America. The imager covers a zenith angle range of about $80^{\circ}$, while the receiver collects signals for the elevation angle greater than $15^{\circ}$.

$\left(47.9^{\circ} \mathrm{W}, 16^{\circ} \mathrm{S} ;-9.4^{\circ} \mathrm{N}\right.$ geomagnetic). The overlapped coverage provides scientists with a good opportunity to understand the relationship between ionospheric plasma bubbles and irregularities over Brazil (see Fig. 1). In this paper, a simultaneous observation of the two instruments is examined. The two long-term databases are then employed to investigate the occurrence of ionospheric plasma bubbles and irregularities in various solar activities, seasons, and local times.

\section{Observation and interpretation}

Several photometers and imagers were operated at Cachoeira Paulista during 1977-1998 while a ground-based GPS receiver has been set up at Brasilia since 1995. Figure 1 illustrates the locations of the imagers, and the receiver, as well as the associated view area and encompassed coverage superposed on the map of South America. The imager covers a zenith angle range of about $80^{\circ}$, while the GPS receiver collects signals for the elevation angle greater than $15^{\circ}$. Because the OI6300 ̊ emission layer is assumed to be at the height of the $270 \mathrm{~km}$ (Sobral et al., 2002) and the ionospheric-point height of GPS satellite-receiver path is given to be $400 \mathrm{~km}$ (cf. Tsai and Liu, 1999), the scanning photometers at Cachoeira Paulista observe bubbles in a range of about $13^{\circ} \mathrm{S}$ to $32^{\circ} \mathrm{S}$ latitudes $\left(-7^{\circ} \mathrm{N}\right.$ to $-25^{\circ} \mathrm{N}$, geomagnetic) and the ground-based GPS receiver at Brasilia monitors bubbles in a range about $7^{\circ} \mathrm{S}$ to $24^{\circ} \mathrm{S}$ latitudes $\left(-2^{\circ} \mathrm{N}\right.$ to $-17^{\circ} \mathrm{N}$, geomagnetic). The corresponding horizontal-coverage diameters of the two observations are both about $2000 \mathrm{~km}$, and their overlapped coverage is in a range of about $13^{\circ} \mathrm{S}$ to $24^{\circ} \mathrm{S}$ latitudes $\left(-7^{\circ} \mathrm{N}\right.$ to $-17^{\circ} \mathrm{N}$, geomagnetic).

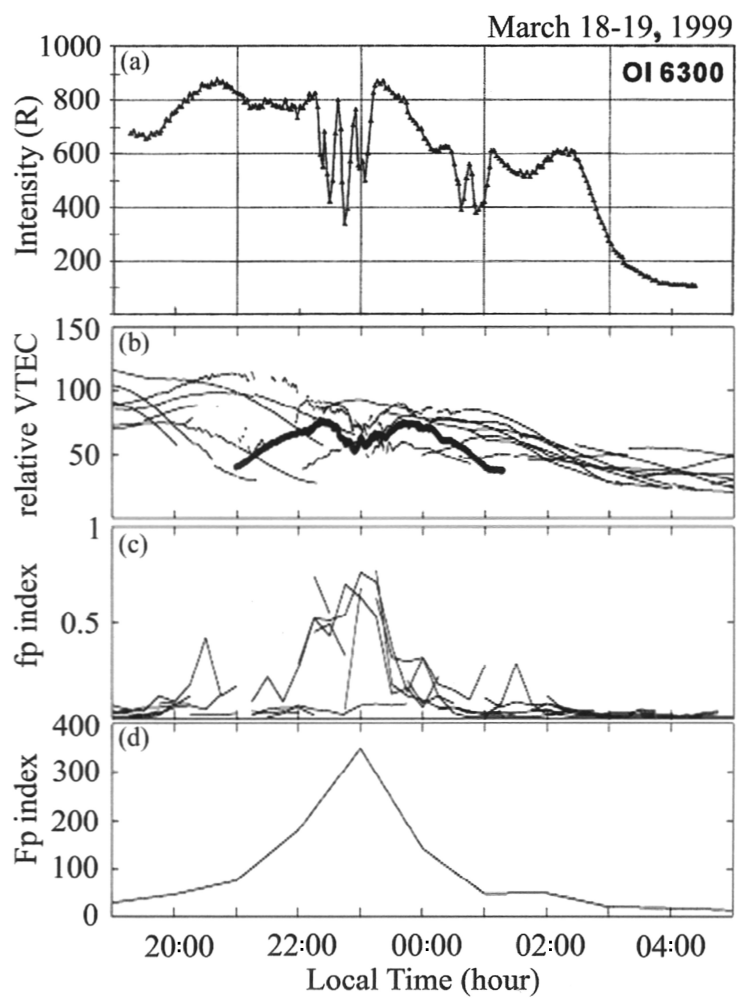

Fig. 2. The nocturnal ionospheric phenomena recorded with a photometer and a GPS receiver on 18 March 1999. (a) Intensity variations of OI6300 F-region component observed by a zenith photometer over Cachoeira Paulista. After Takahashi et al. (2001). (b) Relative vertical TEC derived from GPS at Brasilia, (c) 15-min $f p$ index, and (d) hourly $F p$ index.

A simultaneous observation using the two instruments was carried out from the evening of 18 March 1999 to the predawn of 19 March 1999. Figure 2a, adopted from Takahashi et al. (2001), illustrates absolute values of the emission rate of $630 \mathrm{~nm}$, in Rayleighs, over Cachoeira Paulista, observed with a zenith looking airglow imager during the occurrence of the plasma bubble event. It can be seen that large and small intensity depletions occur from 22:10 to 23:10 LT and from 00:20 to 01:05 LT, respectively. To quantitatively characterize phase fluctuations, we derive the relative vertical total electron content (VTEC), as well as calculate the indices $f p$ and $F p$ from the high-pass-filtering GPS slantTECs (for details, see Mendillo et al., 2000). The index $f p$ of a certain GPS satellite $n$ is defined as the median value over a 15-min period of phase fluctuation data from a GPS satellite,

$f p(n, h, i)=\operatorname{Median}\left|\frac{\Delta \mathrm{TEC}}{\min }\right|$,

where $\triangle$ TEC is defined by subtracting each TEC from its previous value, and $i$ denotes each quarter of the hour, $h$. These $f p$ values are then averaged over a period of $1 \mathrm{~h}$ for all satellites available to a station, and multiplied by 1000 , to 


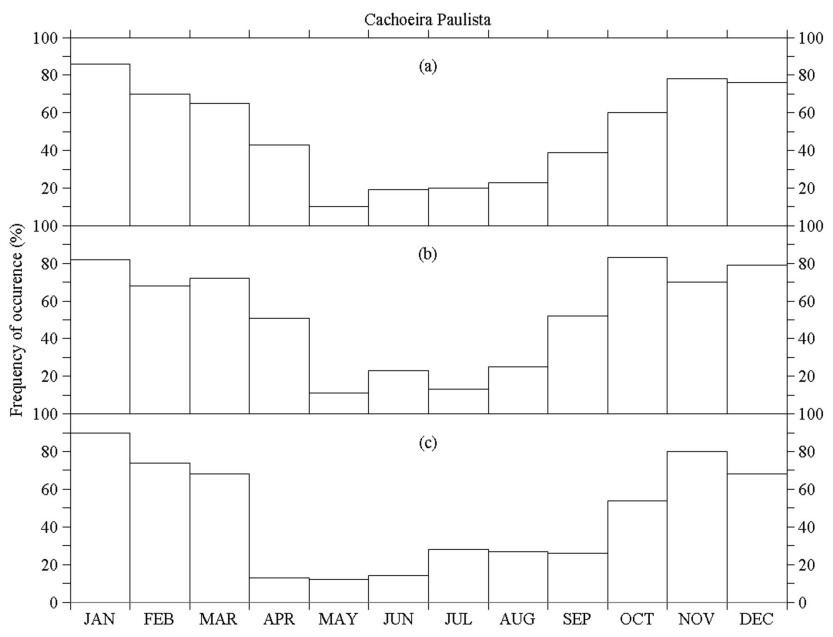

Fig. 3. Frequency of occurrence of the plasma bubbles over Cachoeira Paulista. After Sobral et al. (2002). (a) complete solar cycle (1977-1998, 934 days), (b) high solar activity (1978-1982, 19881992, 442 days), and (c) low solar activity (1977, 1983-1987 and 1993-1998, 492 days).

give the integer index $F p$, which represents the average level of irregularities in the hour above that specific station,

$F p(h)=\frac{\sum_{n}^{\mathrm{nsat}}\left(\sum_{i}^{k} f p(n, h, i) / k\right)}{\operatorname{nsat}(h)} \times 1000$.

Figures $2 \mathrm{~b}, 2 \mathrm{c}$, and $2 \mathrm{~d}$ illustrate the corresponding VTEC, $f p$ and $F p$ indices, respectively. It can be seen that when the large depletions in the emission rate occur from 22:10 to 23:10 LT (Fig. 2a), the decreases in VTEC (Fig. 2b) and the significant enhancements of the two indices (Figs. $2 \mathrm{c}$ and 2d) also appear during 22:00-23:30 LT. The heavy curve in Fig. 2b illustrates that the VTEC measured using the GPS satellite PRN\#4 decreases, which agrees well with the OI6300 A depletions in Fig. 2a. However, there are no obvious GPS VTEC decreases and phase fluctuations observed during the small intensity depletions appearing from 00:20 to 01:05 LT.

To further investigate ionospheric plasma bubbles and irregularities occurring in various solar activities, the longterm database of the photometer obtained at Cachoeira Paulista is compared with the GPS phase fluctuations recorded at Brasilia. Figure 3, adopted from Sobral et al. (2002), illustrates the frequency of bubble occurrence in data from 934 days of experimental observations of the atomic oxygen 630-nm nocturnal airglow by means of scanning photometers and imagers during the period of 19771998. The frequency of occurrence yields greater values in October-March but smaller values in April-September during the 22 years (Fig. 3a). For the high solar activity period the frequency of occurrence produces greater values in September-April but smaller values in May-August (Fig. 3b). Similarly, for the low solar activity period the frequency of occurrence produces greater values in OctoberMarch but smaller values in April-September (Fig. 3c). Note

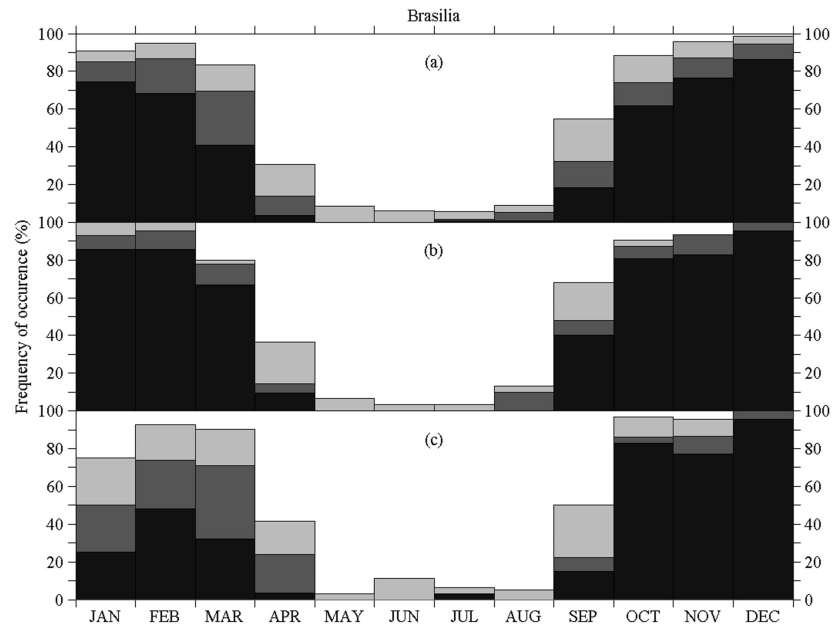

Fig. 4. Frequency of occurrence of the ionospheric irregularities over Brasilia (gray, dark, and black denote the frequency proportions of $F p>50,100$, and 200, respectively): (a) half solar cycle (1995-2000), (b) high solar activity (solar maximum, 2000), and (c) low solar activity (solar minimum, 1997).

for the summer months (November-February) of peak occurrence rate that there is little difference in the occurrence frequency between solar maximum and minimum. Likewise, the occurrence frequencies for the winter months (MayAugust) also show no clear dependence on the solar activity. On the other hand, notable dependence of bubble development on solar activity is evident during the equinoctial months (March-April, September-October).

Due to the availability of the GPS data recorded at Brasilia, the occurrence of ionospheric irregularity is investigated only from 1995 to the present. Mendillo et al. (2000) suggest that the background noise level of irregularities has an $F p$ value of $<50$, an $F p$ value between 50 and 200 representing the existence of moderate irregularities, and $F p>200$ indicates the occurrence of very strong irregularity levels. For a detailed study, the appearance proportions of $F p>50,100$ and 200 (gray, dark, and black in Fig. 4) observed during nighttime (between 18:00 and 06:00 LT) are employed to evaluate the frequency of occurrence of the ionospheric irregularities during the observation period of 1995-2000, the high solar (maximum 2000), and low (minimum 1997) activity periods. Except in January of the high solar activity periods, occurrence frequencies in Figs. 4a, 4b, and 4c for the $F p>100$ are similar to those in Figs. 3a, 3b, and 3c, respectively.

The advantage of the GPS observation is that the two indices can be continuously obtained. This further allows us to investigate the diurnal variations of GPS phase fluctuations in various months during 1995-2000. Figures 5b and 5d illustrate that the occurrence rate of phase fluctuations is substantial low in June but considerable high in December, respectively. Nevertheless, no obvious solar activity dependences can be found in either June or December. However, the occurrence rate of phase fluctuations generally increases with solar activity in March (Fig. 5a) and September (Fig. 5c), 


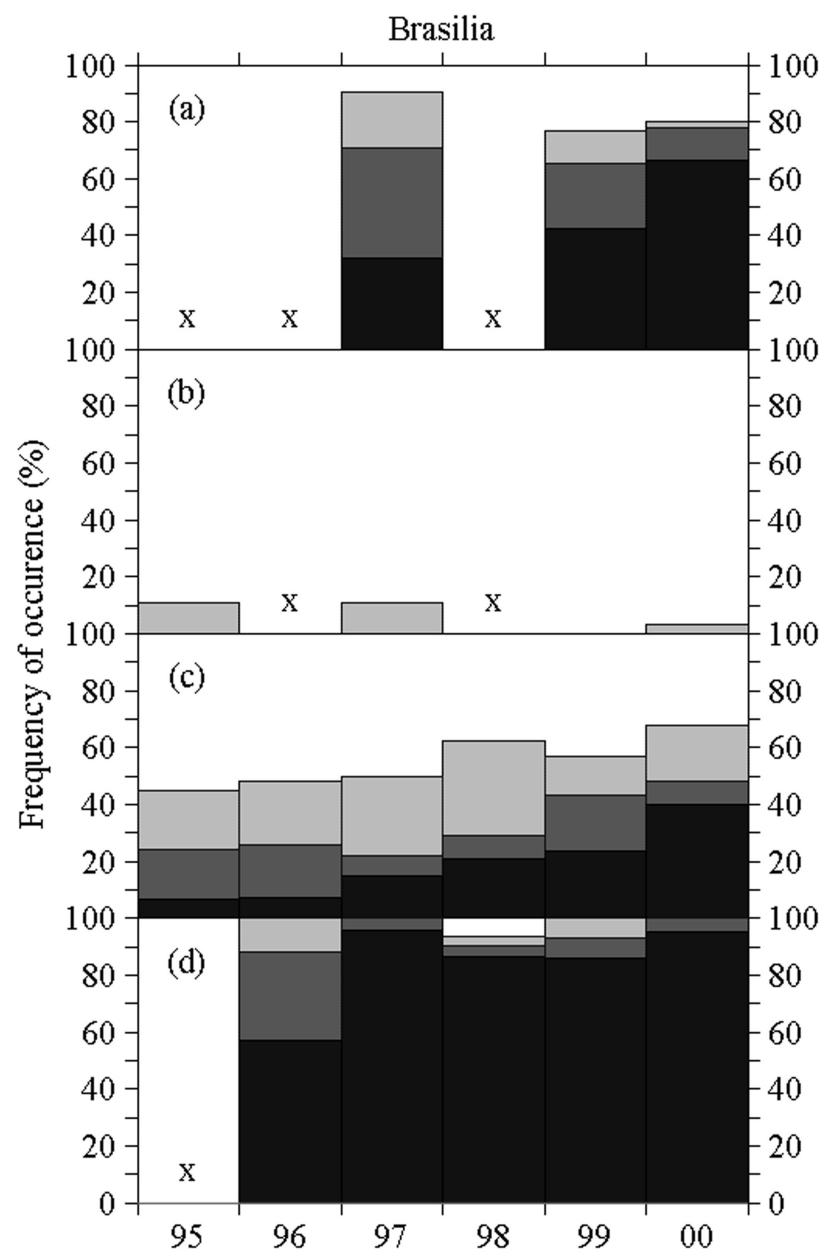

Fig. 5. The solar activity variations of GPS phase fluctuations in different months during 1995-2000 over Brazil (gray, dark, and black denote the frequency of occurrence of $F p>50,100$, and 200, respectively): (a) March, (b) June, (c) September, and (d) December. The cross symbols denote missing data.

especially for stronger irregularities (for example, $F p>100$ ). Figure 6 displays that the irregularity levels are generally below the noise level $F p<50$ between May and August (low irregularity months), and are very strong $F p>200$ between September and April (high irregularity months). Meanwhile, it can be seen that the $F p$ yield maximum values during daytime (about 12:00 or 14:00 LT) of the low irregularity months, and during nighttime (20:00-01:00 LT) of the high irregularity months.

\section{Discussion}

It is known that the photometers, imagers, and GPS measurements observe the large-scale phenomena of airglows, plasma depletion, and TEC phase fluctuations (Mendillo et al., 2000; Sobral et al., 2002; Takahashi et al., 2001; Aarons et al., 1997). The long-term studies of airglows and GPS phase fluctuations have been separately shown in the early papers (e.g. Sobral et al., 2002; Mendillo et al., 2000; Shan et al., 2002). In this work, we examined simultaneous observation of the two instruments and presented the statistical characteristics of airglows and GPS phase fluctuations during solar minimum and maximum.

The simultaneous observations of the imager and GPS ground-based receiver during 18-19 March 1999 show that the large intensity depletions and phase fluctuations appear between 22:10 and 23:10 LT. However, no obvious phase fluctuations could be observed during the occurrence of the small depletions between 00:20 and 01:05 LT. Note in this simultaneous study that the observations of the imager were obtained around the zenith while the phase fluctuations were derived from all the GPS satellites, moving in various directions at various locations, within the field-of-view of the receiver. In general, bubbles appear in the western sky as an elongated north-south strip of reduced airglow brightness which moves eastward, passing through the local zenith. When a strip concurrently moves into the zenith of the imager and some line-of-sights between the GPS satellites and the receiver, the two observations agree with each other. The heavy curve in Fig. 2b illustrates the VTEC variations when the GPS satellite PRN\#4 moved approximately along the longitude $45^{\circ} \mathrm{W}$, which has the same magnetic flux tube of the zenith of the imager. It might be that either bubbles or irregularities in the moving strip were too small and insignificant to be detected by the GPS observation and/or the strip was not simultaneously observed by the two instruments, which result in the discrepancy that occurred between 00:20 and 01:05 LT. To answer this, instead of single receiver, the GPS phase fluctuation study might need a network with several scattered receivers to have better coverage. Nevertheless, the large intensity depletions and phase fluctuations concurrently detected between 22:10 and 23:10 LT suggest that observations of the collocated photometer and GPS receiver can be employed to statistically study the climatology of ionospheric plasma bubbles and irregularities.

The comparison of the two climatology results shows that except in January of the low solar activity periods, occurrence frequencies of the bubbles observed by the imagers and photometers, and those of the index $F p>50$ derived from the GPS receiver, are similar. A detailed study reveals that using the imager results as references, the frequencies of occurrence of $F p>50$ are slightly larger during May-August but somewhat smaller during October-March. The discrepancies between the two climatology studies might result from the two different database periods (imagers/photometers: 19771998; GPS: 1995-2000) and/or limited data being used to calculate the occurrence frequencies. Take the discrepancy in January of the solar minimum shown in Figs. $3 \mathrm{c}$ and $4 \mathrm{c}$ as an example. The frequencies of occurrence of various $F p$ are simply derived from 11-day GPS data in January 1997. Thus, the number of data points could significantly affect the correctness of the occurrence frequencies. Nevertheless, either possibility shall eventually be answered when two common long-term databases become available.

Meanwhile, it can be found from the imager and GPS results that the seasonal patterns of the bubble and irregularity 

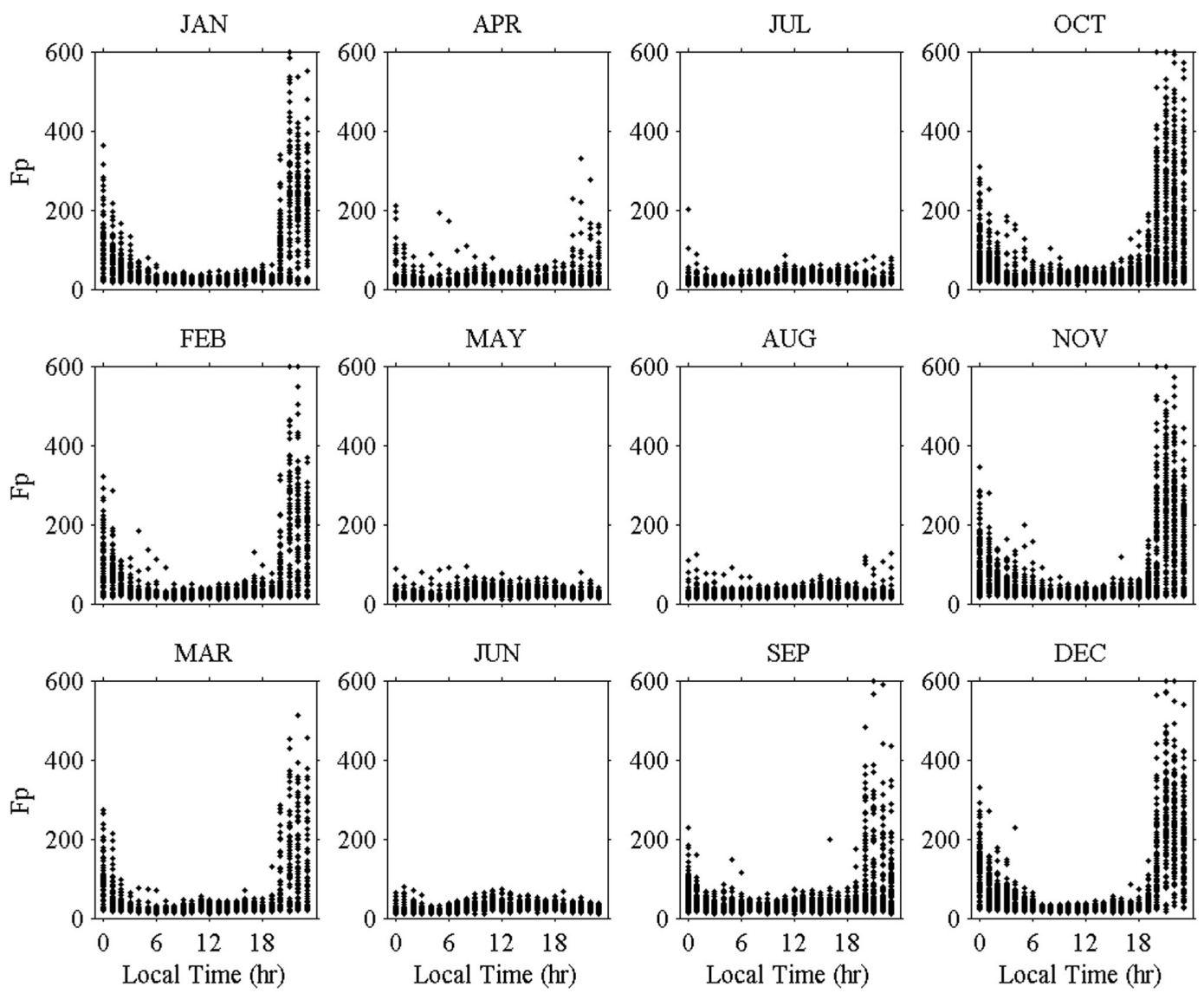

Fig. 6. The diurnal variations of GPS phase fluctuations in various months during 1995-2000 over Brazil.

occurrences are similar for both high and low solar activity during November-February of the local winter months (see Figs. 3b, 3c, 4b, 4c, and 5d), however, with an increase in solar activity during March-April and September-October of the equinoctial months (Figs. 3b, 3c, 4b, 4c, 5a, and 5c). It is interesting to find that based on ionograms over Cachoeira Paulista recorded in the October months of 1973-1983 Abdu et al. (1985) conclude that the occurrence of spread-F is proportional to the solar activity. Sahai et al. (1999) reported that the occurrences of plasma depletions are relatively smaller during low solar activity, than high solar activity by using an eight-year imager data set observed over Cachoeira Paulista (March 1987-October 1991 and September 1994-January 1998). Their results generally agree with Abdu et al. (1985), Sobral et al. (2002), and the results reached in this paper during the equinoctial months.

\section{Conclusions}

This work is the first attempt to make a comparison between the climatology of ionospheric plasma bubbles and GPS phase fluctuations in the Brazilian sector. The two occurrence frequencies concurrently increase with solar activity during the equinoctial month, with yield maximum values in the local summer and minimum values in winter, and a pronounced appearance between 20:00 and 01:00 LT in October-March. The agreements in the two statistical results indicate that the GPS observations provide alternative and useful approaches to further understand climatology of the ionospheric plasma bubbles and irregularities.

Acknowledgements. The GPS data are obtained from the international GPS Service (IGS). This research was supported by the Ministry of Education under grant 91-N-FA07-7-4 for the iSTEP project to National Central University.

Topical Editor M. Lester thanks two referees for their help in evaluating this paper.

\section{References}

Aarons, J.: The longitudinal morphology of equatorial F-layer irregularities relevant to their occurrence, Space Sci. Rev., 63, 209-243, 1993.

Aarons, J., Mendillo, M., and Yantosca, R.: GPS phase fluctuations in the equatorial region during sunspot minimum, Radio Sci., 32, 1535-1550, 1997.

Abdu, M. A., Sobral, J. H. A., Nelson, O. R., and Batista, I. S.: Solar cycle related range type spread-F occurrence characteristics over equatorial and low latitude station in Brazil, J. Atmos. Terr. Phys., 47, 901-905, 1985.

Kelley, M. C.: The Earth's Ionosphere, Academic Press, San Diego, 487, 1989. 
Mendillo, M., Lin, B., and Aarons, J.: The application of GPS observations to equatorial aeronomy, Radio Sci., 35, 885-904, 2000.

Sahai, Y., Fagundes, P. R., and Bittencourt, J. A.: Solar Cycle Effects on Large Scale Equatorial F-Region Plasma Depletions, Adv. Space Res., 24, 1477-1480, 1999.

Shan, S. J., Liu, J. Y., Kuo, F. S., Liu, C. C., and Tsai, H. F.: GPS TEC phase fluctuations observed along the America Sector during low irregularity activity months of 1997-2000, Earth, Planets, and Space, 54, 141-152, 2002.

Sobral, J. H. A., Abdu, M. A., and Batista, I. S.: Airglow studies on the ionosphere dynamics over low latitude in Brazil, Ann. Geophysicae, 36, 2, 199-204, 1980a.

Sobral, J. H. A., Abdu, M. A., Batista I. S., and Zamlutti, C. J.: Association between plasma bubble and airglow disturbances over Brazilian low latitudes, Geophys. Res. Lett., 11, 980-982, 1980 b.

Sobral, J. H. A., Abdu, M. A., Takahashi, H., Sawant, H. S., Zamlutti, C. J., and Borba, G. L.: Solar and geomagnetic activity effects on nocturnal zonal velocities of ionospheric plasma depletions, Adv. Space Res., 24, 11, 1507-1509, 1999.

Sobral, J. H. A., Abdu, M. A., Takahashi, H., Taylor, M. J., de Paula, E. R., Zamlutti, C. J., de Aquion M. G., and Borba, G.L.: Ionospheric plasma bubble climatology over Brazil based on 22 years (1977-1998) of $630 \mathrm{~nm}$ airglow observations, J. Atmo. Solar Terr. Phys., 64, 1517-1524, 2002.
Taylor, M. J., Eccles, J. V., Labelle, J., and Sobral, J. H. A.: Highresolution OI $(630 \mathrm{~nm})$ image measurements of F region depletion drifts during the Guará campaign, Geophys. Res. Lett., 24, 1699-1702, 1997.

Takahashi, H., Taylor, M. J., Sobral, J. H. A., Medeiros, A. F., Gobbi, D., and Santana, D. C.: Fine structure of the ionospheric plasma bubbles observed by the OI 6300 and 5577 airglow images, Adv. Space Res., 27, 1189-1194, 2001.

Tsai, H. F. and Liu, J. Y.: Ionospheric total electron content response to solar eclipses, J. Geophys. Res., 104, 12 657-12 668, 1999.

Valladares, C. E., Sheehan, R., Basu, S., Kuenzler, H., and Espinoza, J.: The multi-instrumented studies of equatorial thermosphere aeronomy scintillation system: Climatology of zonal drifts, J. Geophys. Res., 101, 26 839-26850, 1996.

VanZandt, T. E. and Peterson, V. L.: Detailed maps of tropical nightglow enhancements and their implications on the ionospheric F2 Layer, Ann. de Géophys., 24, 747-749, 1968.

Weber, E. J., Buchau, J., Eather, R. H., and Mende, S. B.: Northsouth aligned equatorial airglow depletions, J. Geophys. Res., 83, 712-716, 1978. 